\title{
鼎湖山异龄马尾松针叶长度序列元素分布
}

\author{
旷远文 ${ }^{1,2}$ 温达志 ${ }^{*}$ 周国逸 ${ }^{1}$ 张德强 ${ }^{1}$ \\ （1 中国科学院华南植物园,广州 510650）（2 中国科学院研究生院, 北京 100039）
}

摘 要 利用植物体元素化学分析结果来诊断环境污染对森林健康影响和监测环境污染程度已成为诸多生态学 家和环境学者广为采用的方法之一。该研究选择广东肇庆鼎湖山健康马尾松 (Pinus massoniana) 前年生针叶和当年

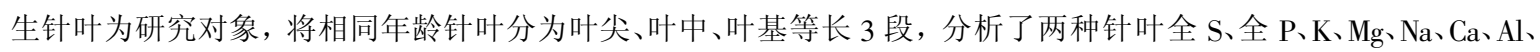
$\mathrm{Mn} 、 \mathrm{Zn} 、 \mathrm{Cu} 、 \mathrm{Fe} 、 \mathrm{~Pb} 、 \mathrm{Cr} 、 \mathrm{Cd}$ 和 Ni15 种元素及相应 $\mathrm{Ca} / \mathrm{Al}$ 值在叶尖、叶中部、叶基部和叶鞘的分布模式。结果表明: 前 年生针叶元素平均值除全 $\mathrm{S} 、$ 全 $\mathrm{P} 、 \mathrm{~K}$ 和 $\mathrm{Cd}$ 外, 其它元素浓度都高于当年生针叶, $\mathrm{Ca} / \mathrm{Al}$ 值则是当年生针叶小于前 年生针叶, 表明当年生针叶受 $\mathrm{Al}$ 毒大于前年生针叶; 在针叶长度序列不同部位间, 元素分布不均匀, 全 S、Na、Ca、 $\mathrm{Al}$ 和 $\mathrm{Mn}$ 在两种针叶长度序列上没有显著性差异, $\mathrm{K} 、 \mathrm{Mg} 、 \mathrm{Zn} 、 \mathrm{Fe} 、 \mathrm{Cr} 、 \mathrm{Cd} 、 \mathrm{Ni}$ 和 $\mathrm{Ca} / \mathrm{Al}$ 值差异均达显著水平, 而全 $\mathrm{P} 、$ $\mathrm{Cu} 、 \mathrm{Cd}$ 和 $\mathrm{Pb}$ 仅在前年生针叶不同部位间差异显著; 针叶 $\mathrm{Al}$ 浓度和 $\mathrm{Ca} / \mathrm{Al}$ 值都表明马尾松已经处于严重 $\mathrm{Al}$ 毒胁迫 下; 两种针叶各部位 $\mathrm{Fe} 、 \mathrm{Zn}$ 和 $\mathrm{Cu}$ 浓度远超出该地区马尾松元素的背景值, 暗示马尾松针叶已受到严重重金属毒 害; 马尾松叶鞘 $\mathrm{Fe} 、 \mathrm{Cu} 、 \mathrm{Zn} 、 \mathrm{~Pb} 、 \mathrm{Cd} 、 \mathrm{Ni}$ 和 $\mathrm{Cr}$ 含量显著高于针叶其它部位, 表明叶鞘能累积环境重金属, 可以作为马 尾松在污染环境下遭受重金属危害指标加以利用, 是一种有广泛应用前景的生物指示物。该研究所采用的技术和 方法对环境监测, 尤其是对利用生物化学方法评价环境污染对森林健康影响评价体系的完善和生物监测指标的利 用具有指导意义, 可为今后评估相似环境污染地区森林健康和树木正常生长提供参考。

关键词 化学分析 元素分布 针叶鼎湖山 马尾松

\section{DISTRIBUTION OF ELEMENTS ALONG THE LENGTH OF DIFFERENT- AGED NEEDLES OF PINUS MASSONIANA AT DINGHUSHAN}

\author{
KUANG Yuan-Wen ${ }^{1,2}$ WEN Da-Zhi ${ }^{1 *}$ ZHOU Guo-Yi ${ }^{1}$ and ZHANG De-Qiang ${ }^{1}$ \\ (1 South China Botanical Garden, Chinese Academy of Sciences, Guangzhou 510650, China) \\ (2 Graduate School of Chinese Academy of Sciences, Beijing 100039, China)
}

\begin{abstract}
Chemical analysis of plant tissues, such as in pine trees, is a frequently used method to evaluate the changes of forest health caused by air pollution. Pine needles also have been used widely as bioindicators of atmospheric pollution due to their wide distribution and easy identification. However, the results of whole needle analysis may ignore differences in elemental concentrations in particular needle parts. To date, only limited research has described the concentrations of different elements in various parts of needles injured by pollutants.

Masson pine ( Pinus massoniana) is a pioneer species widely spread throughout Southern China. Six separate trees were selected from Dinghushan, Guangdong Province and cut down. The healthy looking current year $(\mathrm{C})$ and previous year $(\mathrm{C}+1)$ needles were separated from branches at the upper, middle and lower crown. The needles were cut into three sections, defined as tip section $(\mathrm{T})$, middle section $(\mathrm{M})$ and base section (B), proportional to the needle length. The sheath (S) of each needle-age group also was collected. All parts of the different-aged needles were dried and ground for chemical analysis of the elements, total $\mathrm{S}$, total P, K, Mg, Na, Ca, Al, Mn, Zn, Cu, Fe, Pb, Cr, Cd and Ni. The Ca/Al ratios of each needle component were calculated.

The mean concentrations of the elements in the $\mathrm{C}+1$ needles were higher than in the $\mathrm{C}$ needles, except for total $\mathrm{S}$, total $\mathrm{P}, \mathrm{K}$ and $\mathrm{Cd}$ which were not significantly different, whereas the $\mathrm{Ca} / \mathrm{Al}$ ratios were lower. The elements were unevenly distributed along the length of the needles. The total $\mathrm{S}, \mathrm{Na}, \mathrm{Ca}, \mathrm{Al}$ and $\mathrm{Mn}$ did not show significant differences among the different needle sections whereas $\mathrm{K}, \mathrm{Mg}, \mathrm{Zn}, \mathrm{Fe}, \mathrm{Cr}$, Ni and the $\mathrm{Ca} /$ $\mathrm{Al}$ ratios differed statistically among the different sections of the $\mathrm{C}$ and $\mathrm{C}+1$ needles. The total $\mathrm{P}, \mathrm{Cu}, \mathrm{Cd}$ and
\end{abstract}

基金项目: 国家自然科学基金项目(30370283)、中国科学院知识创新工程重要方向性项目 (SCX2-SW-120)、广东省自然科学基金项目 (04002306)和佛山科技发展基金项目 
$\mathrm{Pb}$ were significantly different among the sections in the $\mathrm{C}+1$ needles only. The element patterns along the needles were mainly caused by air pollution. The needles were under $\mathrm{Al}$ stress as determined by the $\mathrm{Al}$ concentrations and $\mathrm{Al} / \mathrm{Ca}$ ratios, especially in the base section. Values of $\mathrm{Al}$ and/or $\mathrm{Ca} / \mathrm{Al}$ ratios in the base of the needle potentially can be used as an early diagnostic index of $\mathrm{Al}$ toxicity. Concentrations of $\mathrm{Cu}$ and $\mathrm{Pb}$ in the area were far above background values for Masson Pine needles implying that excessive heavy metals might damage the needles. Concentrations of $\mathrm{Fe}, \mathrm{Cu}, \mathrm{Pb}, \mathrm{Zn}, \mathrm{Cd}, \mathrm{Ni}$ and $\mathrm{Cr}$ in the needle sheath were significantly higher than in the other three sections of the needle suggesting that the needle sheath might be a better bioindicator of those elements than other needle parts. The results of this study and the techniques employed constitute a new contribution to the development of biogeochemical methods for environmental monitoring. These methods may be of value for follow up studies aimed at the assessment of municipal and industrial pollution.

Key words Chemical analysis, Element distribution, Needle parts, Dinghushan, Pinus massoniana

大气污染和气候变化是威胁全球森林健康持续 发展的两个最主要因子, 据估计, 到 2050 年全球受 S 沉降胁迫森林面积将达到 $59 \times 10^{5} \mathrm{~km}^{2}, 2100$ 年全球 将有 $49 \%$ 的森林受到 $\mathrm{O}_{3}$ 危害, 因而很有必要重新 评估大气污染对森林健康的监测方法 (Percy \& Ferretti，2004）。基于环境污染对生态系统负面影响日 益严重,利用植物体化学分析的结果来诊断环境污 染和监测环境的变化已成为诸多生态学家和环境学 者广泛采用的方法之一。苔藓目前已被当成环境生 物指示物广泛使用 (Svoboda et al., 2000; Figueira et $a l ., 2002$ )。松类, 尤其是欧洲赤松 (Pinus sylvestris) 因为分布广泛, 是国外学者广为应用于监测大气污 染对森林健康影响的高等指示植物 (Kurczynska et al., 1997; Ewa et al., 1997; Gietych et al., 1997; Yilmaz \& Zengin, 2004), 针叶大量元素和微量元素浓 度可以用于评估森林遭受大气污染损害程度 (Dmuchowski \& Bytnerowicz, 1995; Shparyk \& Parpan, 2004）。然而, 由于多数研究是将整条针叶元素平均 浓度作为环境污染程度指标加以利用, 其结果必然 掩盖针叶出现明显受害症状前元素在针叶中的累积 分布状况, 尤其是忽略了元素在针叶不同部位 (长度 序列上) 的浓度差异, 对选择针叶合适部位作为生物 指示物显然存在不足。同时, 分析针叶不同部位元 素浓度分布模式, 还可以为研究者提供污染环境中 针叶营养状态等信息。目前仅 Jamrich ( 1972) 和 Giertych 等(1997) 研究了污染条件下欧洲赤松针叶 长度序列上不同部位元素分布模式及其浓度差 异。

我国珠江三角洲地区近几十年经济快速发展, 环境污染也日趋严重, 给当地植被和生态系统带来 了严重损害 (温达志等, 2003), 大气污染引起森林 健康衰退已有报道 (严昌荣和朱忠保, 1996; 包维 楷, 1999; 骆土寿等, 2001)。本研究选取广泛分布
于我国南方的马尾松 (Pinus massoniana) 异龄针叶, 以鼎湖山为研究地点, 通过分析针叶长度序列上不 同部位元素浓度, 旨在探讨: 1) 在当前大气污染水平 下马尾松异龄针叶长度序列不同元素分布特征;2) 污染条件下马尾松针叶是否出现营养元素缺乏或重 金属元素累积; 3 ) 针叶化学元素分析作为生物指示 物的可行性及其最适部位。研究结果以期为当前污 染水平下鼎湖山南亚热带季风常绿阔叶林健康评价 体系构建和管理提供科学依据, 也为我国其它相似 污染区域森林健康与否的诊断和环境监测生物途径 的改进提供参考。

\section{1 材料和方法}

\section{1 样品采集与处理}

2002 年 12 月于鼎湖山自然保护区五棵松针阔 混交林选择 6 株马尾松, 样树表观健康, 无病虫害, 无断梢, 树高 $15 \mathrm{~m}$ 左右, 树龄约 40 年。样树之间相 距至少 $100 \mathrm{~m}$ 且远离道路。样树伐倒后在东西南北 方位上中下树冠分别均匀采摘当年 (2002 年)生针 叶和前一年(2001 年)生针叶, 每株树异龄针叶各 3 个重复。针叶采摘后立即分别装入塑料袋密封带回 实验室, 不加任何清洗, 用不锈钢刀片将异龄针叶分 别逐条切为等长 3 段 (针尖段、中间段和针基段), 将 同龄针叶相同部位混合, 同时分别小心采摘异龄针 叶叶鞘。切分后的样品均在 $65{ }^{\circ} \mathrm{C}$ 下烘干 $24 \mathrm{~h}$, 经微 型植物粉碎机磨碎后装瓶备化学分析。另取少量异 龄完整针叶, 分离叶鞘后烘干, 分别粉碎, 测针叶和 叶鞘各元素平均含量。

\section{2 化学分析}

所有元素化学分析过程均参照中国生态系统研 究网络观测分析标准(董鸣, 1996)进行。植物全 S 和全 $P$ 用国产分光光度计测定; $\mathrm{K} 、 \mathrm{Mg} 、 \mathrm{Ca} 、 \mathrm{Na} 、 \mathrm{Cu}$ 、 $\mathrm{Fe} 、 \mathrm{Zn} 、 \mathrm{Mn} 、 \mathrm{Ni}$ 用澳大利亚产原子吸收光度计 (AAS, 
GBC932AA)测定, Cd 和 Cr 用德国产石墨炉原子吸 收光度计 (GFAS, ZEENIT60)测定, $\mathrm{Al}$ 和 $\mathrm{Pb}$ 用美国产 等离子耦合发射光谱( ICP PS-1000AT) 测定。测定 时用元素标准溶液 (由日本 KAWO 化学工业有限公 司提供)配置系列浓度, 绘制工作曲线。各工作曲线 相关系数 $\left(R^{2}\right)$ 均大于 0.999 。

\section{3 统计分析}

数据用 SPSS 11.0 软件进行多因素方差分析, 并对实验结果进行成对样本检验, 以比较同龄针叶 不同部位间元素的差异性,显著性水平为 0.05 。

\section{2 实验结果}

\section{1 异龄针叶及其叶鞘元素平均含量及 $\mathrm{Ca} / \mathrm{Al}$ 值}

从表 1 中可以发现, 前年生针叶元素含量除全 $\mathrm{S} 、$ 全 $\mathrm{P} 、 \mathrm{~K}$ 和 $\mathrm{Cd}$ 平均值显著低于当年生针叶含量 外, 其余元素都表现出前年生针叶含量大于当年生 针叶含量的特征, 一些重金属如 $\mathrm{Mn} 、 \mathrm{Zn} 、 \mathrm{Fe} 、 \mathrm{~Pb}$ 和 $\mathrm{Cr}$ 在前年生针叶中的平均值分别是当年生针叶平均值 的 1.2、2.1、2.0、1.6 和 1.6 倍, 而 $\mathrm{Mg} 、 \mathrm{Ca}$ 和 $\mathrm{Al}$ 则分 别为 $1.4 、 2.7$ 和 1.9 倍, 显示了这些元素在老叶中 的累积效应, $\mathrm{Na}$ 和 $\mathrm{Cu}$ 在两种针叶的平均值没有显 著统计差异。就 $\mathrm{Ca} / \mathrm{Al}$ 值来看, 两种针叶平均值差 异显著, 当年生针叶低于前年生针叶, 显示出当年生 针叶所受到 $\mathrm{Al}$ 毒大于前年生针叶。

全 $\mathrm{S} 、 \mathrm{~K} 、 \mathrm{Mn}$ 和 $\mathrm{Ca} / \mathrm{Al}$ 在当年生叶鞘中的平均值 要高于其在前年生针叶平均值, $t$ 检验表明, $\mathrm{K}$ 和
$\mathrm{Mn}$ 浓度在两种叶鞘中差异不显著, Ca、 $\mathrm{Al} 、 \mathrm{Zn} 、 \mathrm{Cu}$ 、 $\mathrm{Fe} 、 \mathrm{~Pb} 、 \mathrm{Cr}$ 和 $\mathrm{Ni}$ 在当年生叶鞘的浓度均显著低于前 年生叶鞘, 说明叶鞘能有效累积这些元素。

\section{2 针叶长度序列上元素分布特征}

各元素在不同年龄针叶长度序列上的分布模式 如图 1 和图 2。同龄针叶 4 个部位间元素浓度差异 性以不同字母标识在图中。总 $\mathrm{S}$ 含量在异龄针叶长 度序列上的分布模式存在差异。前年生针叶 $\mathrm{S}$ 含量 从叶尖 $(T)$ 到叶鞘 $(S)$ 部分呈缓慢增加趋势, 当年生 针叶各部位全 S 含量均高于前年生针叶相应部位, 表现出中部浓度最低后线性上升特征。老叶各区分 段总 $\mathrm{S}$ 含量介于 $0.03 \% \sim 0.05 \%$ 之间, 当年生针叶 不同区分段总 $\mathrm{S}$ 含量介于 $0.04 \% \sim 0.11 \%$ 之间, 但 两种针叶 4 个部位全 $\mathrm{S}$ 含量差异均不显著。当年生 针叶全 $\mathrm{P}$ 含量最大值出现在叶基部, 叶尖、叶中部和 叶鞘部位全 $\mathrm{P}$ 含量处于相近水平。前年生针叶叶 尖、叶中部和叶基部全 $\mathrm{P}$ 含量均低于当年生针叶相 同部位, 但叶鞘部位全 $\mathrm{P}$ 含量与当年生叶鞘相等, 显 示叶鞘部位全 $\mathrm{P}$ 含量可能与叶龄无关。统计检验表 明, 全 $\mathrm{P}$ 在前年生针叶 4 个部位中叶鞘含量显著高 于其它 3 个部位, 而在当年生针叶中不存在这种差 异。前年生针叶长度序列上 $\mathrm{Al}$ 浓度高于当年生叶 对应部位。前年生针叶中, Al 浓度在叶尖-叶中_叶 基序列上缓慢线性下降, 到叶鞘骤然升高, 当年生针 叶 $\mathrm{Al}$ 浓度在叶中部稍微降低后上升, 且在叶鞘没有 骤然升高。从统计结果看, 不论当年生针叶还是前年

表 1 马尾松异龄针叶及叶鞘元素平均含量 \pm 标准差和 $\mathbf{C a} / \mathbf{A l}$ 值

Table 1 Mean $\pm S D$ values of elements and $\mathrm{Ca} / \mathrm{Al}$ ratio in the whole needles

\begin{tabular}{|c|c|c|c|c|}
\hline \multirow{2}{*}{$\begin{array}{c}\text { 元素 } \\
\text { Element }\end{array}$} & \multicolumn{2}{|c|}{ 当年生叶 Current year } & \multicolumn{2}{|c|}{ 前年生叶 Previous year } \\
\hline & 针叶 Needle & 叶鞘 Sheath & 针叶 Needle & 叶鞘 Sheath \\
\hline $\mathrm{S}$ & $0.05 \pm 0.02$ & $0.16 \pm 0.11$ & $0.04 \pm 0.02^{* *}$ & $0.05 \pm 0.02^{* *}$ \\
\hline $\mathrm{P}$ & $0.06 \pm 0.01$ & $0.05 \pm 0.02$ & $0.03 \pm 0.00^{* *}$ & $0.05 \pm 0.02$ \\
\hline $\mathrm{K}$ & $2005.8 \pm 743.3$ & $2256.9 \pm 953.7$ & $1074.5 \pm 789.8^{* *}$ & $1816.2 \pm 351.0$ \\
\hline $\mathrm{Mg}$ & $937.4 \pm 252.2$ & $1090.5 \pm 225.5$ & $1348.0 \pm 566.8^{* *}$ & $1578.9 \pm 365.2$ \\
\hline $\mathrm{Na}$ & $295.1 \pm 53.7$ & $295.2 \pm 57.0$ & $305.5 \pm 66.9$ & $322.4 \pm 56.2$ \\
\hline $\mathrm{Ca}$ & $1465.1 \pm 743.5$ & $1315.3 \pm 753.4$ & $3982.7 \pm 587.2^{* *}$ & $2801.7 \pm 60.6^{*}$ \\
\hline $\mathrm{Al}$ & $280.8 \pm 133.7$ & $537.6 \pm 90.7$ & $540.7 \pm 231.7^{*}$ & $1517.6 \pm 504.1^{*}$ \\
\hline Mn & $313.2 \pm 155.2$ & $333.4 \pm 69.1$ & $370.9 \pm 192.4$ & $279.1 \pm 78.1$ \\
\hline $\mathrm{Zn}$ & $34.5 \pm 10.2$ & $54.6 \pm 8.3$ & $76.7 \pm 25.1^{* *}$ & $116.3 \pm 26.7^{* *}$ \\
\hline $\mathrm{Cu}$ & $15.9 \pm 5.0$ & $18.1 \pm 4.5$ & $16.0 \pm 9.2$ & $57.5 \pm 12.8^{* *}$ \\
\hline $\mathrm{Fe}$ & $143.6 \pm 54.8$ & $458.0 \pm 204.9$ & $284.9 \pm 99.8^{* *}$ & $1063.3 \pm 337.4^{* *}$ \\
\hline $\mathrm{Pb}$ & $2.57 \pm 1.73$ & $5.9 \pm 2.6$ & $4.13 \pm 2.10^{*}$ & $12.6 \pm 3.2^{* *}$ \\
\hline $\mathrm{Cr}$ & $2.32 \pm 1.42$ & $4.71 \pm 0.48$ & $3.73 \pm 1.60^{* *}$ & $20.2 \pm 11.2^{*}$ \\
\hline $\mathrm{Cd}$ & $0.17 \pm 0.15$ & $0.32 \pm 0.09$ & $0.11 \pm 0.04$ & $0.43 \pm 0.16$ \\
\hline $\mathrm{Ni}$ & $0.30 \pm 0.21$ & $0.30 \pm 0.08$ & $0.30 \pm 0.22^{* *}$ & $1.27 \pm 0.19^{* *}$ \\
\hline $\mathrm{Ca} / \mathrm{Al}$ & $5.89 \pm 2.80$ & $3.35 \pm 2.11$ & $8.53 \pm 4.17^{* *}$ & $2.09 \pm 0.43^{* *}$ \\
\hline
\end{tabular}



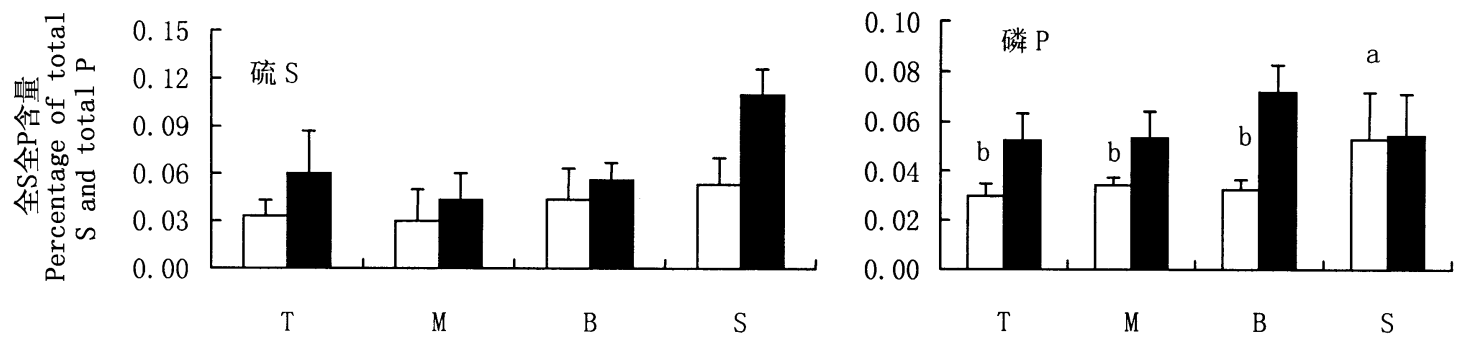

图 1 马尾松当年生针叶 $(\square)$ 和前年生针叶 $(\square)$ 各部位全 $\mathrm{S}$ 和全 $\mathrm{P}$ 百分含量

Fig. 1 The percentages of total S and total P along the current year needle ( $\square$ ) and the previous year needle $(\square)$ of Pinus massoniana, respectively 同龄组针叶不同部位间元素含量差异显著时以不同字母表示 Different letters represent the significant difference among the sections of the same age class needles $(p=0.05, n=6)$ T: 叶尖 Needle tip M: 叶中部 Middle section B: 叶基部 Base section S: 叶鞘 Sheath

生针叶, 叶鞘 $\mathrm{Al}$ 浓度均显著高于其它 3 个部位, 针 叶长度序列上 $\mathrm{Al}$ 浓度没有显著差异性。 $\mathrm{Mg}$ 浓度在 前年生针叶长度序列上呈线性增加, 而在当年生针 叶呈 $\mathrm{V}$ 型分布, 到叶鞘部位两种年龄针叶 $\mathrm{Mg}$ 浓度 均有所下降, 4 个部位 $\mathrm{Mg}$ 浓度差异显著。除叶尖 外, 前年生针叶相同部位 $\mathrm{Mg}$ 浓度均高于当年生针 叶。 $\mathrm{K}$ 浓度与 $\mathrm{Al} 、 \mathrm{Mg}$ 不同, 当年生针叶相同部位 $\mathrm{K}$ 浓度均高于前年生针叶, 但其浓度在当年生针叶长 度序列上没有明显分布规律, 前年生针叶长度序列 上从叶尖到叶鞘部位出现良好的线性上升趋势。两 种针叶不同部位 $\mathrm{K}$ 浓度差异显著。当年生和前年 生针叶长度序列上 $\mathrm{Na}$ 浓度不存在统计性差异, 相同 部位其浓度基本接近, 都处在 $300 \mathrm{mg} \cdot \mathrm{kg}^{-1}$ 左右水平 上。Ca 在两种针叶不同部位分布模式和 $\mathrm{Al}$ 相同, 均表现为前年生针叶大于当年生针叶, 两种年龄针 叶不同部位 $\mathrm{Ca}$ 浓度差异均不显著。在叶尖、叶中和 叶基上, 前年生针叶 $\mathrm{Ca}$ 浓度呈现 $\mathrm{V}$ 型分布, 而当年 生针叶则呈直线下降, 无论是前年生针叶还是当年 生针叶, 最高 $\mathrm{Ca}$ 浓度均出现在叶尖, 而最低 $\mathrm{Ca}$ 浓度 均出现在叶鞘。就 $\mathrm{Ca} / \mathrm{Al}$ 值而言, 两种针叶的最低 比值出现在叶鞘部位, 而最大值在当年生针叶是叶 尖, 前年生针叶是叶基, 4 个部位间 $\mathrm{Ca} / \mathrm{Al}$ 值在前年 生针叶中表现显著差异, 在当年生中叶尖与叶中部、 叶基部和叶鞘间差异不显著(图 2)。Fe 元素在两种 针叶长度序列上的分布模式几乎完全相同, 叶尖、叶 中和叶基部 $\mathrm{Fe}$ 浓度几乎不存在波动现象(前年生针 叶约在 $280 \mathrm{mg} \cdot \mathrm{kg}^{-1}$, 当年生针叶约在 $140 \mathrm{mg} ・$ $\mathrm{kg}^{-1}$ ), 而在叶鞘部位两种针叶都表现出明显 $\mathrm{Fe}$ 累 积(前年生针叶叶鞘 $\mathrm{Fe}$ 浓度达到 $1064 \mathrm{mg} \bullet \mathrm{kg}^{-1}$, 当 年生针叶叶鞘为 $458 \mathrm{mg} \cdot \mathrm{kg}^{-1}$ ), 叶鞘 $\mathrm{Fe}$ 浓度均高出 其它部位 4 倍以上。 $\mathrm{Cu}$ 浓度在两种针叶长度上也
没有太大波动, 都处在 $15 \mathrm{mg} \bullet \mathrm{kg}^{-1}$ 的水平上, 仅在前 年生针叶叶鞘出现 $\mathrm{Cu}$ 累积, 比其它部位浓度高出 3 倍。当年生针叶不同部位 $\mathrm{Cu}$ 浓度没有统计性差异, 但前年生针叶, 尤其是叶鞘部位能有效累积 $\mathrm{Cu}$ 。

痕量金属元素 $\mathrm{Pb}$ (前年生针叶在 $0.4 \sim 12.6$ $\mathrm{mg} \bullet \mathrm{kg}^{-1}$ 之间, 当年生针叶在 $0.3 \sim 5.9 \mathrm{mg} \bullet \mathrm{kg}^{-1}$ 之 间)和 $\mathrm{Cr}$ (前年生针叶在 $2.9 \sim 20.2 \mathrm{mg} \mathrm{kg}^{-1}$ 之间, 当 年生针叶在 $1.2 \sim 4.7 \mathrm{mg} \bullet \mathrm{kg}^{-1}$ 之间)在两种针叶长 度序列上的分布模式非常相似, 即在叶尖、叶中和叶 基部位没有浓度上的差别, 仅在叶鞘部位出现累积, 其浓度是针叶其它部位浓度的 10 倍以上。 Cd 在两 种针叶长度序列上也出现同 $\mathrm{Pb}$ 和 $\mathrm{Cr}$ 相同的浓度分 布, 累积 $\mathrm{Cd}$ 最多的部位都是叶鞘。 $\mathrm{Ni}$ 在两种针叶 长度上虽然没有明显的分布规律, 但不同部位其浓 度差异均达到显著水平(图 2)。

\section{3 讨 论}

由于元素背景值在不同区域存在差异, 本研究 结果还不足以判断某种元素是否过量或不足, 但我 们发现前年生针叶总 $\mathrm{S}$ 含量降低, 可能的原因是随 着针叶衰老, $S$ 会随着其它可移动元素流失 (Rautio \& Huttunen, 2003)。由于 $\mathrm{S}$ 浓度在两种针叶序列上 差异都不显著, 因此难以确定用哪一龄针叶哪部分 作为 $\mathrm{S}$ 危害或 $\mathrm{S}$ 不足的生物指示器。除 $\mathrm{Mg}$ 元素外, 造成 $\mathrm{K}$ 含量在两种针叶长度上不同部位之间的显 著差异可能是由于 $\mathrm{K}$ 的移动性而造成的 (Helmisaari, 1992), 而 Ca 由于在植物体内的移动性 较差, 它们在两种针叶长度上都没有显著差异, 前年 生针叶相同部位 $\mathrm{Ca}$ 浓度高于当年生针叶, 是因为 $\mathrm{Ca}$ 在老叶中会累积。

$\mathrm{Al}$ 浓度在两种针叶叶尖、叶中部和叶基部之间 


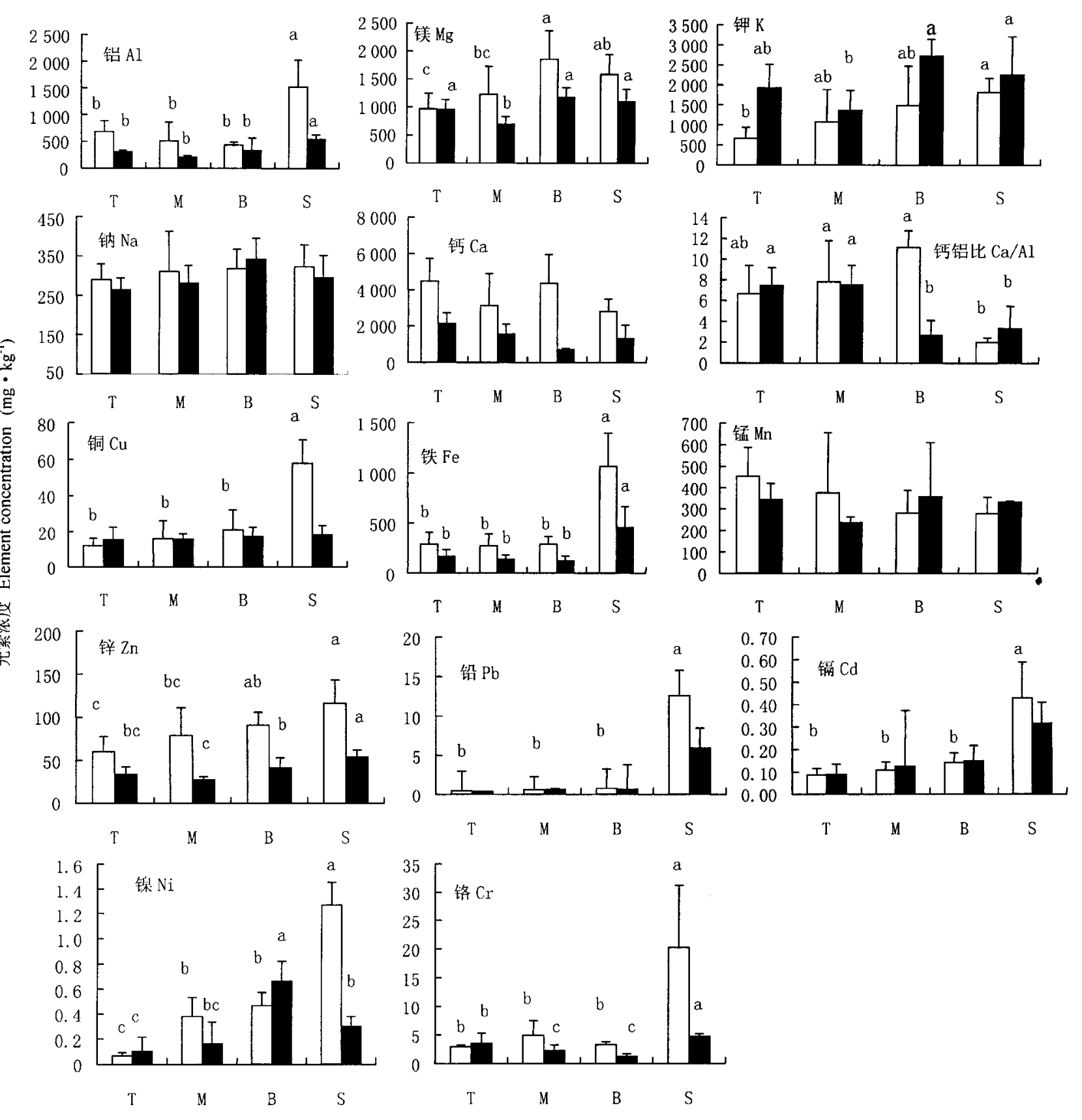

图 2 马尾松当年生针叶 $(\boldsymbol{\square})$ 和前年生针叶 $(\square)$ 各部位元素浓度格局

Fig.2 The distributing patterns of elements along the current year $(\square)$ and previous year $(\square)$ needles of Pinus massoniana, respectively 同龄组针叶不同部位间元素含量差异显著时以不同字母表示 Different letters represent the significant difference among the sections of the same age class needles $(p=0.05, n=6) \quad \mathrm{T}, \mathrm{M}, \mathrm{B}, \mathrm{S}$ : 同图 1 See Fig. 1

不存在显著的差异性。当年生针叶 $\mathrm{Al}$ 浓度最高的 部位是叶基部, 可能与该段比其它叶段幼嫩有关。 叶基部位对 $\mathrm{Al}$ 毒更为敏感, 可以用来早期诊断针叶 受 $\mathrm{Al}$ 胁迫的程度。从 $\mathrm{Ca} / \mathrm{Al}$ 值来看, 在当年生针叶 上,叶尖、叶中、叶基和叶鞘 4 个部位分别为 7.5、 $7.5 、 2.7$ 和 3.3 , 前年生针叶对应值分别为 6.1 、. 7.8 、
11.1 和 2.0, 都低于目前欧洲所认同的 $\mathrm{Al}$ 毒标准值 $\mathrm{Ca} / \mathrm{Al}=12.5$ (Rengel, 1992)。但该标准是否也适用 于亚洲, 还有待于进一步从植物形态和生理生态等 方面进行研究和认定。

不可移动元素 $\mathrm{Fe}$ 浓度无论是在老叶还是新叶 长度序列上没有什么明显差异, 但 $\mathrm{Fe}$ 在不同部位的 
浓度(当年生针叶为 $121.8 \sim 457.9 \mathrm{mg} \bullet \mathrm{kg}^{-1}$, 前年生 针叶为 $274.8 \sim 1063.3 \mathrm{mg} \bullet \mathrm{kg}^{-1}$ ) 远远超过了广州市 马尾松针叶的背景值 $\left(97.6 \mathrm{mg} \cdot \mathrm{kg}^{-1}\right.$ ) (李健和郑春 江,1989), 表明该地区马尾松针叶已遭受到严重的 $\mathrm{Fe}$ 毒害, $\mathrm{Fe}$ 在马尾松叶鞘中的显著累积可以将叶鞘 作为 $\mathrm{Fe}$ 的生物指示物。我们发现, 尽管 $\mathrm{Cu}$ 浓度在 马尾松针叶长度序列除前年生叶鞘外没有显著差 异, 但两种针叶 $\mathrm{Cu}$ 浓度 (当年生针叶为 $15.2 \sim 18.1$ $\mathrm{mg} \bullet \mathrm{kg}^{-1}$, 前年生针叶为 $\left.12.0 \sim 57.5 \mathrm{mg} \bullet \mathrm{kg}^{-1}\right)$ 均显 著高于该区域 $\mathrm{Cu}$ 在马尾松中的背景值 $(11.0 \mathrm{mg}$ ・ $\mathrm{kg}^{-1}$ )(李键和郑春江, 1989), 揭示该地区马尾松已 经受到 $\mathrm{Cu}$ 危害。叶鞘能指示环境中 $\mathrm{Cu}$ 浓度。本研 究发现 $M n$ 在针叶长度序列上不存在显著差异, 当 年生针叶不同部位浓度为 $237.9 \sim 358.2 \mathrm{mg} \bullet \mathrm{kg}^{-1}$, 前年生针叶为 $279.1 \sim 453.4 \mathrm{mg} \cdot \mathrm{kg}^{-1}$, 且远低于该 区域 $\mathrm{Mn}$ 在马尾松的背景值 $\left(977.5 \mathrm{mg} \bullet \mathrm{kg}^{-1}\right.$ ) (李键 和郑春江, 1989), 表明在该区域马尾松存在明显的 $\mathrm{Mn}$ 不足, 可以用来作为针叶健康与否的诊断指标。 $\mathrm{Zn}$ 在两种针叶长度序列上的显著差异是由 $\mathrm{Zn}$ 在针 叶中的移动造成的 (Helmisaari, 1992), 本研究发现 $\mathrm{Zn}$ 从叶尖到叶鞘序列上表现出逐渐上升趋势, 当年 生针叶为 $27.5 \sim 54.6 \mathrm{mg} \cdot \mathrm{kg}^{-1}$, 前年生针叶为 60.2 $\sim 116.3 \mathrm{mg} \bullet \mathrm{kg}^{-1}$, 当年生针叶处于其在该区域的正 常范围 (40.0 mg $\mathrm{kg}^{-1}$ ) (李键和郑春江, 1989), 但老 叶表现出明显的 $\mathrm{Zn}$ 毒害, 表明目前该地区 $\mathrm{Zn}$ 能在 马尾松针叶中显著累积, 其叶鞘也能有效指示环境 中 $\mathrm{Zn} 。 \mathrm{~Pb}$ 是一种大气颗粒污染物, 我们发现, 马尾 松两种针叶长度序列上 $\mathrm{Pb}$ 浓度虽然非常低, 但在前 年生针叶和当年生针叶叶鞘部位, $\mathrm{Pb}$ 浓度分别达到 12.6 和 $6.0 \mathrm{mg} \cdot \mathrm{kg}^{-1}$, 产生这种现象的原因可能是 叶鞘对大气 $\mathrm{Pb}$ 沉降的吸附累积, 表明在该地区大气 沉降产生的 $\mathrm{Pb}$ 量维持在较高水平, 从另一个方面也 启示我们可以用针叶叶鞘 $\mathrm{Pb}$ 含量来指示大气 $\mathrm{Pb}$ 沉 降。

环境中 $\mathrm{Cd}$ 主要来源于人为排放和污染, 极易被 植物根系吸收并在植物体内转移。我们发现, 当年 生针叶序列上 $\mathrm{Cd}$ 浓度为 $0.09 \sim 0.28 \mathrm{mg} \bullet \mathrm{kg}^{-1}$, 前年 生针叶为 $0.08 \sim 0.43 \mathrm{mg} \cdot \mathrm{kg}^{-1}$, 表明工业排放引起 的 Cd 污染可能会对马尾松生长存在潜在危害, Cd 在叶鞘也有累积现象, 也可以作为 $\mathrm{Cd}$ 污染的生物指 示物。本研究发现两种针叶各部位 Ni 浓度前年生 叶鞘 $\mathrm{Ni}$ 浓度显著高于其它部位, 说明叶鞘能指示环 境中的 $\mathrm{Ni}$, 前年生叶鞘 $\mathrm{Cr}$ 也是如此。叶鞘对重金属 的累积, 可能的原因在于: 叶鞘较叶体粗䊁, 表面阻
力大, 能有效吸附重金属; 叶鞘分泌的物质能束缚污 染物; 叶鞘的束状结构能防止雨水对污染物的淋洗 而流失。在生物指示物方面, 叶鞘就有广泛应用前 景。

如上所述, 元素在马尾松异龄针叶长度序列上 分布是不均匀的, 某些元素在叶鞘部位能出现显著 的累积现象。马尾松针叶不同部位, 尤其是叶鞘化 学分析结果可以作为马尾松在污染环境下遭受 $\mathrm{Cu}$ 、 $\mathrm{Pb}$ 和 $\mathrm{Zn}$ 受害指标加以利用。

$$
\text { 参 考 文 献 }
$$

Bao WK (包维楷) (1999). Degraded features of Pinrus massoniana forest stressed by compound-pollution due to particulartes, $\mathrm{SO}_{2}$ and $\mathrm{NO}_{\mathrm{x}}$. Acta Phytoecologica Sinica (植物生态学报), 23, $501-509$. (in Chinese with English abstract)

Dmuchowski W, Bytnerowicz A (1995). Monitoring environmental pollution in Poland by chemical analysis of Scots pine (Pinus sylvestris L.) needles. Environmental pollution, 87, 87-104.

Dong M (董鸣) (1996). Survey, Observation and Analysis of Terrestrial Biocommunities (陆地生物群落调查观测与分析). Standards Press of China, Beijng, 154 - 159. (in Chinese)

Ewa U, Kurczyńska WD, Wielaw W (1997). The influence of air pollutants on needles and stems of Scots pine (Pinus sylvestris L) trees. Environmental Pollution, 98, 325-334.

Figueira R, Sérgio C, Sousa AJ (2002). Distribution of trace metals in moss biomonitors and assessment of contamination sources in Portugal. Environmental Pollution, 118, 153-163.

Giertych MJ, de Temmerman LO, Rachwol L (1997). Distribution of elements along the length of Scots pine needles in a heavily polluted and a control environment. Tree Physiology, 17, 697703.

Helmisaari HS (1992). Nutrient retranslocation within the foliage of Pinus sylvestris. Tree Physiology, 10, 45-48.

Jamrich V (1972). The localization, accumulation and binding of fluorine from industrial fumes in the tissues of the organs of Pinus sylvestris . Zbornik Vedeckych Prac VSLD vo Zwolene, 14, 89 99.

Kurczynska EU, Dmuchowski W, Wloch W, Bytnerowicz A (1997). The influence of air pollutants on needle and stems of Scots pine (Pinus sylvestris L.) trees. Environmental Pollution, $98,325-334$.

Li J (李健), Zheng CJ (郑春江) (1989). Handbook of Data for Environmental Background Values (环境背景值数据手册). Chinese Environmental Science Press, Beijing, 200 - 216. (in Chinese)

Luo TS (骆土寿), Wu ZM (吴仲民), Xu YG (徐义刚), Zhou GY (周光益), He ZC (何在成) (2001). Preliminary studies on the effect of atmosphere pollution on forest and soil in Pearl River Delta, China. Ecologic Science (生态科学), 20, 11 16. (in Chinese with English abstract) 
Percy KE, Ferretti M (2004). Air pollution and forest health: toward new monitoring concepts. Environmental Pollution, 130, $113-126$.

Rautio P, Huttunen S (2003). Total vs. internal element concentrations in Scot pine needles along a sulphur and metal pollution gradient. Environmental Pollution, 122, 273 - 289.

Rengel Z (1992). Role of calcium in aluminium toxicity. New Phytologist, 121, $499-513$.

Shparyk YS, Parpan VI (2004). Heavy metal pollution and forest health in Ukrainian Carpathians. Environmental Pollution, 130, $55-63$

Svoboda L, Zimmermannová K, Kalac P (2000). Concentrations of mercury, cadmium, lead and copper in fruiting bodies of edible mushrooms in an emission area of a copper smelter and a mercury smelter. Science of Total Environment, 246, 61-67.

Wen DZ (温达志), Kuang YW (旷远文), Liu SZ (刘世忠), Lu YD (陆耀东), Li JL (黎建力) (2003). Vegetation damage by long-term air pollution at a rural site in the Pearl River Delta in South China. Journal of Tropical and Subtropical Botany (热 带亚热带植物学报), 11, 386-392. (in Chinese with English abstract)

Yan CR (严昌荣), Zhu ZB (朱忠保) (1996). Declining of Pinus massoniana in relation to air pollution in Dasheng, Lengshuijiang, Hunan. Acta Phytoecologica Sinica (植物生态学报), 20, 207 - 215. (in Chinese with English abstract)

Yilmaz S,Zengin M (2004). Monitoring environmental pollution in Erzurum by chemical analysis of Scots pine (Pinus sylvestris L) needles. Environmental international, 29, 1041 - 1047. 